\title{
ACCURACY IMPROVEMENT OF A IMMS IN AN URBAN SCENARIO
}

\author{
F. Marotta ${ }^{1}$, C. Achille ${ }^{1}$, G. Vassena ${ }^{2}$, F. Fassi ${ }^{1}$ \\ 1 3D Survey Group, ABC Dep, Politecnico di Milano, Via Ponzio 31, 20133 Milano, Italy. \\ $<$ federica.marotta $><$ cristiana.achille $><$ francesco.fassi $>@$ polimi.it \\ ${ }^{2}$ Department of Civil Engineering, Architecture, Territory, Environment and Mathematics (DICATAM), \\ Università degli Studi di Brescia, Via Branze 43, 25123 Brescia, Italy. \\ giorgio.vassena@unibs.it
}

Commission II

KEY WORDS: SLAM, IMMS, Urban Survey, Mobile Mapping, Wearable Mobile Mapping System, Laser Scanner.

\begin{abstract}
:
Indoor Mobile Mapping Systems (IMMSs) technologies are becoming increasingly popular thanks to the possibility of acquiring a massive amount of 3D data in a fast and effective way in those areas where GNSS signal is unavailable, like urban canyons, densely vegetated areas, underground sites and buildings. They offer an efficient way to produce point clouds but with noticeably lower accuracy than the traditional Terrestrial Laser Scanning (TLS). The present paper wants to analyse two different methods to improve the accuracy of the point cloud coming from an IMMS survey in a vast urban scenario. The first approach uses points collected during an RTK GNSS survey as Ground Control Points (GCPs). The second one involves TLS static scans as Ground Control Scans (GCSs). Both these procedures allow us to introduce constraints before generating the rigid final point cloud. The tested IMMS is the Backpack Heron MS Twin Color, produced by Gexcel S.r.l. The instrument was tested during the acquisition of the historical centre of Meda (MB) in the northern part of Italy. Results show that in those areas between the constraints, the maximum error of residuals on checkpoints is some decimetres. The IMMS has allowed us to quickly survey a vast area not otherwise obtainable with traditional survey techniques. The developed procedures proved to be essential for proper reconstruction of the environment.
\end{abstract}

\section{INTRODUCTION}

Indoor Mobile Mapping Systems (IMMS) technologies are becoming more and more popular thanks to the possibility of acquiring a massive amount of 3D data in a fast and effective way. These techniques can be successfully used in those areas where GNSS signal is unavailable, like urban canyons, densely vegetated areas, underground sites and buildings (Wang et al., 2020). IMMSs consist of three components: a 3D mapping unit, i.e., the sensors acquiring $3 \mathrm{D}$ data, a positioning and navigation unit for spatial referencing and the control unit in charge of combining the information coming from the different sensors (Puente et al., 2013).

The state-of-the-art concerning the MMSs consists mainly in Light Detection And Ranging (LiDAR) sensors as a 3D mapping unit, usually equipped with cameras for getting the RGB information of the scene (Nocerino et al., 2017). Depending on the support on which they are mounted, MMSs can be divided into wearable, handheld, trolley, vehicle-based.

Differently from Mobile Mapping Systems, where the navigation system, including GNSS and an Inertial Measurement Unit (IMU), provides the trajectory and attitude for generating the georeferenced 3D point cloud, IMMSs rely on Simultaneous Localisation And Mapping (SLAM) algorithms. SLAM, initially used in robotics and 3D vision, combines the IMU and scans, acquired and automatically registered along the trajectory, to simultaneously build a map and localise the sensor within the environment almost in real-time (Karam et al., 2019).

IMMS technologies are offering an efficient way to produce point clouds, but with noticeably lower global accuracy than the traditional Terrestrial Laser Scanning (TLS) (Lehtola et al., 2017). The final point cloud accuracy depends on many factors, including the absence of GNSS information aimed at minimising the drift error, the survey design, the geometric properties of the environment and incorrect operator's behaviours during the survey. Moreover, choosing the numerous parameters in the postprocessing phase is equally crucial. All these factors lower the level of reliability and repeatability of the method. To limit this problem, the introduction of geometric constraints inside the SLAM post-processing procedure is mandatory.

The present paper wants to analyse two different methods to improve the accuracy of the point cloud coming from a Backpack Laser Scanner IMMS survey in a vast urban scenario characterised by high-rise buildings and narrow streets. The first approach uses points collected during an RTK GNSS survey as Ground Control Points (GCPs). The second one involves TLS static scans as Ground Control Scans (GCSs). It is essential to highlight that both these procedures allow us to introduce constraints inside the SLAM algorithm before the generation of the rigid final point cloud.

\subsection{Related works}

As previously stated, MMSs drastically reduce the survey time acquisition when dealing with vast areas and complex scenarios to the detriment of accuracies achievable with more classical static type instruments. In Nocerino et al. (2017), two portable MMS are evaluated indoor and outdoor. One instrument has a GNSS receiver, while the other one does not. Check measurements are acquired using a TLS for the indoor test and a van-based MMS for the outdoor one. Another exhaustive indooroutdoor test field is reported in Tucci et al. (2018), where three IMMSs are compared by taking the TLS survey as ground truth. A very complete comparison is reported by Lehtola et al. (2017), where eight different MMSs are tested in three areas. Lagüela et al. (2018) present a wearable prototype of IMMS, developing also a SLAM method to map and generate point clouds.

Results of the reported tests show the potentialities of the instruments when restricted outdoor areas or indoor spaces are surveyed. However, things are different when investigating vast 
regions or the environment characterised by poor geometrical properties (Marotta et al., 2021). Here MMSs show their weakness, introducing errors in the trajectory estimation that cannot be neglected. In a previous experience (Fassi et al. 2019), an extensive total station survey had to be performed to adjust the final trajectory of the Leica Pegasus Backpack used to survey a large wooded flat area. Lucks et al. (2021) use additional information from an a priori known 3D city model, available via geo-web services and a Digital Elevation Model of the surveyed area, to improve the trajectory estimation in an urban environment. Line-feature-based SLAM is implemented in Jung et al. (2015), where a constrained adjustment is incorporated to reduce the uncertainty of line-feature extraction. The adjustment is based on the assumption that the main structures in an indoor environment are formed by parallel or orthogonal lines.

\subsection{Case study}

The Backpack IMMS was tested during the acquisition of the historical centre of Meda (MB) in the northern part of Italy. The interested roads cover a total length of about $2.3 \mathrm{~km}$; they are characterised by a narrow width (on average: $4.5 \mathrm{~m}$ ) and are surrounded by buildings on both sides with heights between 10 and $20 \mathrm{~m}$.

The survey's main goal was to obtain the street plan and facades of the main street, Corso Matteotti, highlighted in blue in Figure 1 , at a scale of 1:200/1:500. The resulting mobile mapping point cloud made it possible to obtain the right and left elevations of Corso Matteotti at 1:200 scale and the 3D model, at the same scale, with the restitution of the shape of the roofs and eaves height, as well as the position of the through openings and courtyards.

The survey provided an excellent opportunity to test different methodologies to increase the accuracy achievable by the instrument in an urban environment.

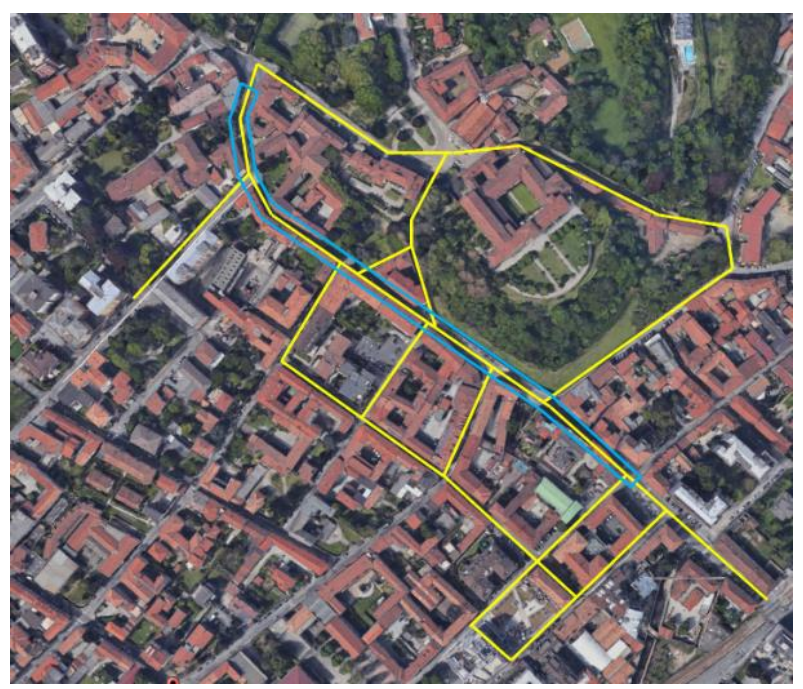

Figure 1. Area interested by the survey in the centre of Meda (MB). Corso Matteotti is highlighted in blue. Trajectories covered by the IMMS are yellow-coloured.

\section{MATERIALS AND METHODS}

The tested IMMS is the Backpack Heron MS Twin Color (Figure 2), produced by Gexcel S.r.l. (Cantoni and Vassena, 2019, Gexcel, 2021). Heron MS Twin Color weights about $5 \mathrm{~kg}$ and is equipped with two Velodyne Puck LITE LiDAR sensors emitting 16 lines each, which results in 600'000 acquired points per second. One sensor rotates on a vertical axis, while the second rotates on a $45^{\circ}$ tilted axis. In this way, the total Field Of View (FOV) is almost $360^{\circ} \times 360^{\circ}$. The maximum range of the sensors is $100 \mathrm{~m}$, and the declared local accuracy is about $3 \mathrm{~cm}$. The instrument is also equipped with a full-resolution RGB camera to acquire images to colour the point cloud. The panoramic camera on top of the pole is characterised by a continuous $15 \mathrm{~Hz}$ acquisition (1920x1080 pixel full HD) and an on-demand image acquisition at $5 \mathrm{k}$ (5640x2820 pixel). An example of an available panoramic image is shown in Figure 3. While walking, it is possible to check in real-time the surveyed environment thanks to a portable tablet that can be carried with a dedicated harness, leaving the hands free. It is also possible to acquire the $5 \mathrm{k}$ images by pressing a dedicated button on the tablet. Although the sensors working time is about 3.5 hours in the continuous acquisition, the limiting factor is attributable to the working time of the tablet, which is about 2 hours.

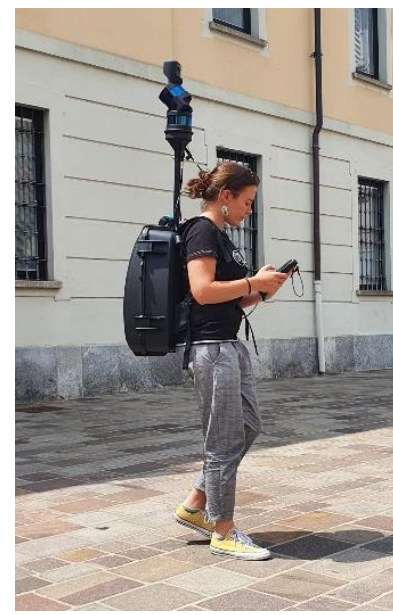

(a)

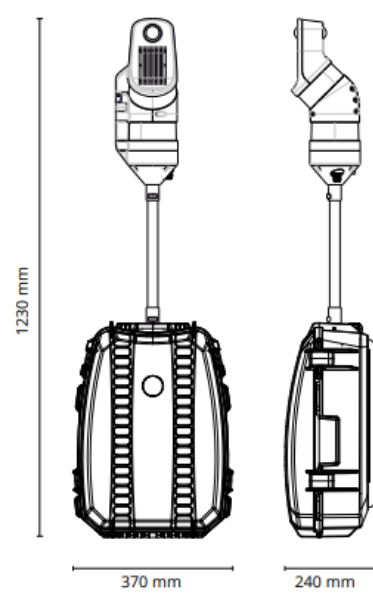

(b)
Figure 2. (a) The operator wearing Backpack Laser Scanner IMMS Heron MS Twin Color produced by Gexcel S.r.l. (BS); (b) Technical drawing of the instrument. Image modified from www.gexcel.it

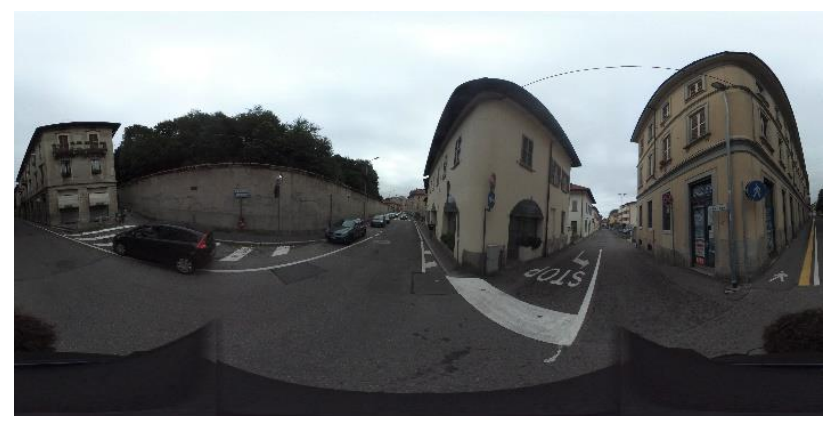

Figure 3. On demand panoramic image obtained by pressing the dedicated button on the touchscreen control unit. Resolution: 5640x2820 pixel.

Five scans were acquired with TLS Leica HDS-7000 at locations considered strategic for the implementation of the developed methodology, i.e. at the border and at the centre of the surveyed area. Finally, a RTK GNSS survey allowed us to obtain 48 welldistributed points to be used both as constraints, check points and to georeference the TLS scan. The accuracy of these points is typical of a RTK survey of $2-3 \mathrm{~cm}$ along $\mathrm{X}$ and $\mathrm{Y}$ direction and 4-5 $\mathrm{cm}$ along $\mathrm{Z}$ direction. 


\subsection{Data acquisition}

Ten trajectories were acquired with Heron MS Twin Color. Every trajectory is a closed-loop to minimise the drifts error that accumulates while walking. Moreover, each of them has a portion that overlaps with at least another one to better register the scan maps in the processing phase.

Moreover, considering one of the main goals of the survey, i.e., the creation of the 3D model, and giving the inclination of the laser beams, the roads were surveyed in both directions to obtain a complete representation of all the environment.

To respect all these pre-requisite, the surveyed path resulted longer than the natural length of the interesting roads. The total duration of the acquisition was 1 hour and 35 minutes, and the total covered path was almost $5.5 \mathrm{~km}$. Details of the trajectories are reported in Table 1.

\begin{tabular}{cccc}
\hline Trajectory & $\begin{array}{c}\text { Length } \\
(\mathbf{m})\end{array}$ & $\begin{array}{c}\text { Duration } \\
(\mathbf{m m : s s})\end{array}$ & N. clouds \\
\hline 1 & 816.50 & $14: 07$ & 8478 \\
2 & 43.11 & $00: 39$ & 392 \\
3 & 742.30 & $13: 32$ & 8121 \\
4 & 441.80 & $07: 25$ & 4458 \\
5 & 567.80 & $10: 56$ & 6569 \\
6 & 294.10 & $05: 36$ & 3367 \\
7 & 689.80 & $11: 03$ & 6631 \\
8 & 651.70 & $11: 06$ & 6661 \\
9 & 555.20 & $09: 13$ & 5531 \\
10 & 687.50 & $11: 28$ & 6881 \\
\hline
\end{tabular}

Table 1. Characteristics of the 10 trajectories performed with Gexcel Heron MS Twin Color.

\subsection{Data processing}

Data acquired with Backpack Heron MS Twin Color were processed with Heron Desktop, proprietary software developed by Gexcel. The workflow is subdivided into 5 steps: Odometer, Create Maps, Global Optimization, Clean Data, Go to Reconstructor.

In the Odometer phase, trajectories are reconstructed one by one. Specifically, it is possible to check the accordance between the alignment of the scans acquired along the trajectory and the information coming from the IMU by selecting the proper parameters made available to the user. Parameters are divided into 3 sections regarding i) the cloud filtering, i.e., the minimum and maximum distance of acquired points from the sensor, ii) the creation of groups of subsequent point clouds to be registered and iii) the registration of these aforementioned groups of point clouds. The operator is helped in interpreting successful operation by the color of the trajectory, which is greener the more agreement there is between scans and IMU (Figure 4). The preview of the raw acquired trajectory may differ from its actual shape as it can happen that the sensors get lost during the acquisition, e.g. due to sudden operator's movements. However, this does not affect at all its final reconstruction.

Once the trajectory is correctly reconstructed, the point clouds continuously acquired during the survey are divided into the socalled "Local Maps", in the second step of the procedure. The idea beyond that phase, which is based on a Gexcel patented algorithm, is to merge clusters of mobile point clouds in a selected range of trajectory to create groups of point clouds Local Maps - that can be managed as static scans. Not all mobile point clouds but only the most robust ones are considered in this process by selecting the proper parameters described as follows. Here again it is possible to select the minimum and maximum distance of acquired points from the sensors and the voxel size, thus setting the resolution of the Local Maps. Additionally, it can be chosen whether to consider data coming from Velodyne sensor 1 and/or Velodyne sensor 2. The length of the local maps is decided, i.e., every how many metres a Local Map is created as well as the overlapping percentage with the next one and the minimum translation and rotation to add a cloud to the current local map. In this way, when the operator stands still for a certain period and the instrument continues recording, redundant data are not taken into account. Finally, feature extractor specific parameters can be set.

As a result of the choice of parameters, 444 Local Maps were created starting from the 57'089 acquired mobile point clouds (sum of column "N. clouds" in Table 1).

In the third step, the Global Optimisation, Local Maps having in common portions of the surveyed environment are then linked together by applying the point-to-point Iterative Closest Point (ICP) algorithm. The algorithm converges to a local minimum by minimising the sum of the squared distances of the corresponding points belonging to two different point clouds. (Wang et al., 2020). After creating all the possible matches, a cluster optimisation is performed. Those links with an error exceeding the desired threshold are removed in an iterative cluster optimisation-removal of matches process. Considering the scale of the requested 3D model and the local accuracy of the instrument, the chosen threshold error was set to $0.10 \mathrm{~m}$. Figure 5 shows the results at the end of the "Global Optimization" step.

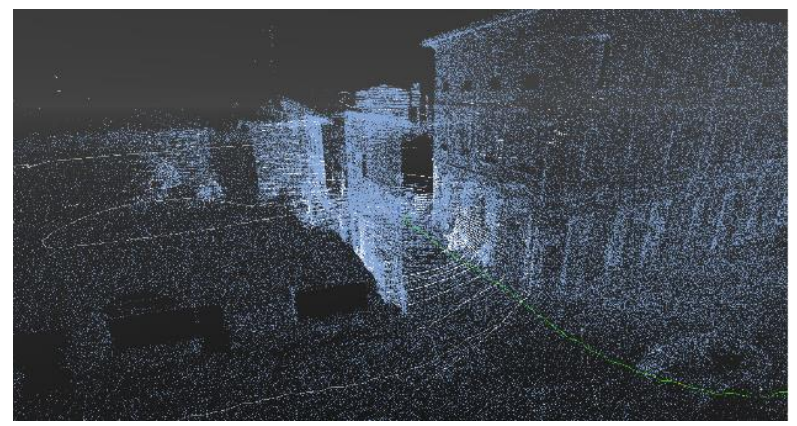

(a)

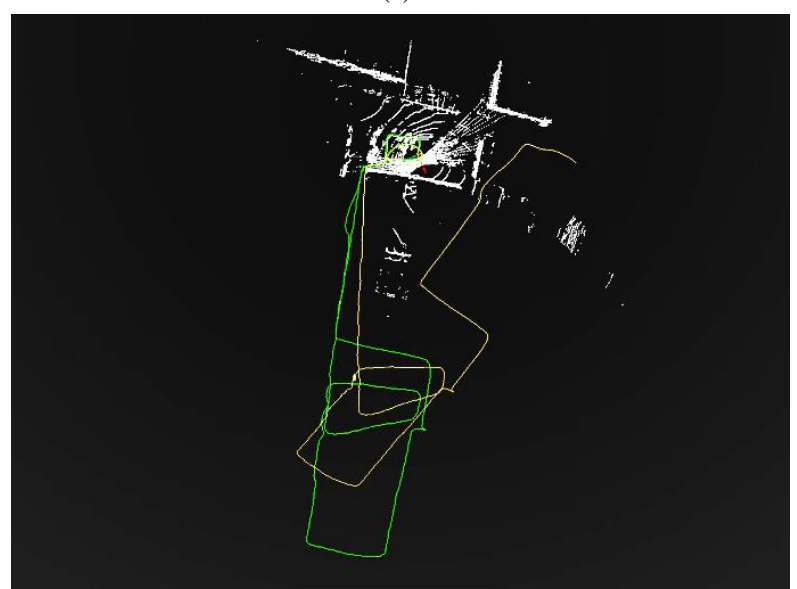

(b)

Figure 4. (a) Trajectory reconstruction during the Odometer phase; (b) Planimetric view of the overlap between the imported raw trajectory (yellow) and the same trajectory after the Odometer phase (green). 


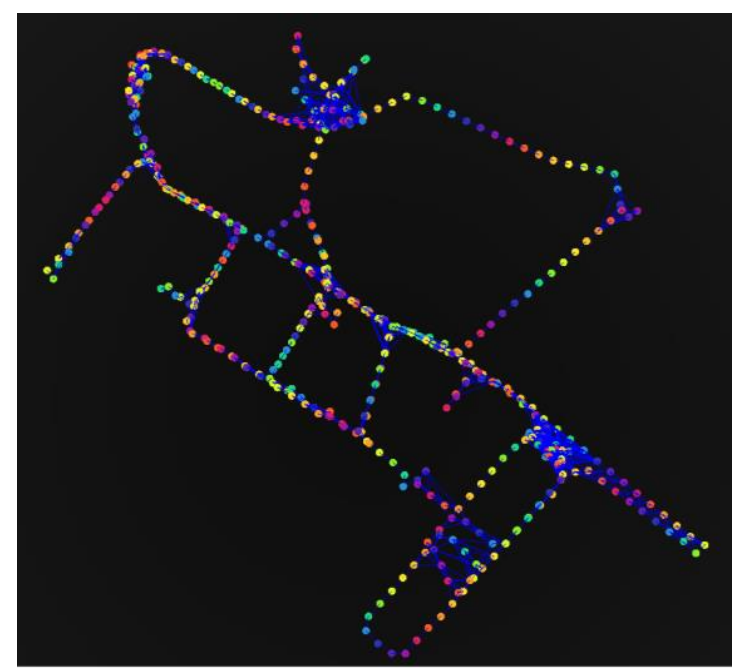

Figure 5. The 444 Local Maps (coloured points) and the 1512 matches (blue segments) generated at the end of the "Global Optimization" phase.

The Clean Data phase is aimed at removing the ghost points, which means those points belonging to moving objects that create wakes, worsening the quality of the final result. Moving objects, in the case at issue, are: pedestrians, cars, vans, motors. To remove ghost points, an explored space analysis is performed by setting the desired range around the sensor to be investigated and a proper time range. Then, subsequent mobile point clouds are analysed: if the point is not present in all the point clouds in the selected time range, it is removed.

The fifth and last step, "Go to Reconstructor", regards the export of the obtained final rigid point clouds, generated by the correctly reconstructed trajectories, to Reconstructor, the other proprietary software developed by Gexcel S.r.l. This step is essential to visualise, edit and finally export the point cloud in formats compatible with other 3D data management software. It is possible to export only data coming from Velodyne sensor 1 and/or Velodyne sensor 2 and data with a sensor inclination of up to a maximum of chosen degrees or even data in the desired range by selecting a minimum and maximum distance around the instrument.

After the export, the import to Reconstructor software follows. The duration of this phase depends on the hardware characteristics. Moreover, it is strongly influenced by the choice of elements to be imported, such as the acquired images - the full $\mathrm{HD}$ and/or the $5 \mathrm{k}$ images - and the chosen voxelisation of the point clouds: MMS point clouds are large-volume and have heavy redundancy and irregular distributions. The voxelisation procedure is crucial to reduce the size of these data and to regularise them. Clearly, the smaller the voxel, the higher the resolution of the point cloud and, consequently, more time is required to import the data. In this step, a set of preprocessing filters like the Statistical Outlier Removal (SOR) can be applied to import only points with the highest confidence. Once imported all the desired trajectories, point clouds can be explored and edited according to the operator's aims.

\subsection{Developed methodology}

The developed methodology focuses on introducing separately points and scans constraints inside the SLAM algorithm before the generation of the rigid final point cloud. This allows to improve the accuracy of the IMMS point cloud and to directly georeference the outcome. The two proposed methods have been designed in response to the mobile point cloud obtained at the end of the Heron Desktop procedure, without introducing any constraints. The point cloud was exported to Reconstructor and georeferenced by means of $3 \mathrm{GCP}$.

The first method involves 22 Ground Control Points (GCPs) obtained with RTK GNSS survey and georeferenced in the Reference System WGS84 UTM 32N. The GCPs are welldistributed all over the entire surveyed area, as shown in Figure 9. The survey time was about 1 hour and a half.

In the Global Optimisation phase, the software gives the possibility to load the highest-resolution version of the local maps on which to precisely identify the GCPs (Figure 6a), assigning the point picking accuracy, named "Heron Accuracy". The list of coordinates was loaded into Heron Desktop, as far as their accuracy: $3 \mathrm{~cm}$ in $\mathrm{X}$ and $\mathrm{Y}, 4 \mathrm{~cm}$ in the $\mathrm{Z}$ direction. For the case at hand, the Heron Accuracy was set to $2 \mathrm{~cm}$ in $\mathrm{X}, \mathrm{Y}$ and $\mathrm{Z}$ directions. The 22 GCPs were then added as constraints, and the cluster optimisation was performed (Figure 6b).
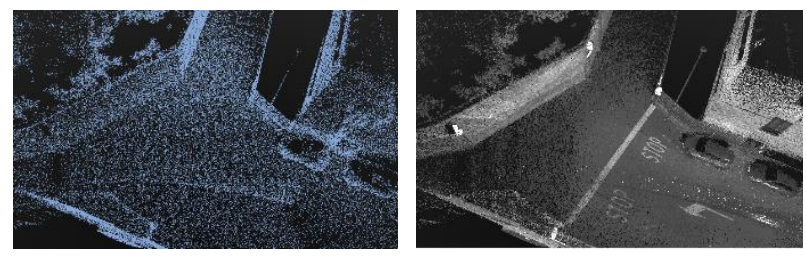

(a)

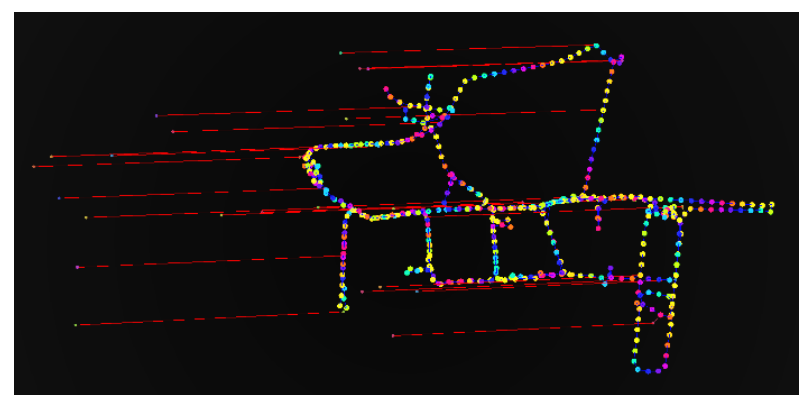

(b)

Figure 6. (a) Comparison between the standard version of Local Maps normally displayed to the user and the high-resolution version loaded for precise detection of GCPs; (b) Matches among GCPs in the reference position and identified on Local Maps.

The second approach is based on the use of Ground Control Scans (GCSs) and implies the use of five scans acquired using TLS Leica HDS-7000, located in the centre and at the area's border (Figure 7). The entire operational time to acquire the scans was about 1 hour and a half. Scans have been georeferenced in the Reference System WGS84 UTM 32N using the RTK GNSS survey points. The idea of using GCSs is due to the high redundancy of the constraint which allows a statistical optimisation of the process. Scans are robust and bind the mobile point cloud in all directions, strongly directing in this way the final trajectory.

The five scans were subsampled to $2 \mathrm{~cm}$ and imported into Heron Desktop software inside the Global Optimisation phase. Here they were locked in the cluster, given their known reference position in space, and linked to the Local Maps overlapping the same areas. As explained in the previous paragraph, the iterative procedure of cluster optimisation-removal of matches was performed, reducing the matches' error to values compatible with the instrument accuracy. Figure 8 shows how the trajectory near GCS 1 changed before and after the introduction of the GCS constraint. 


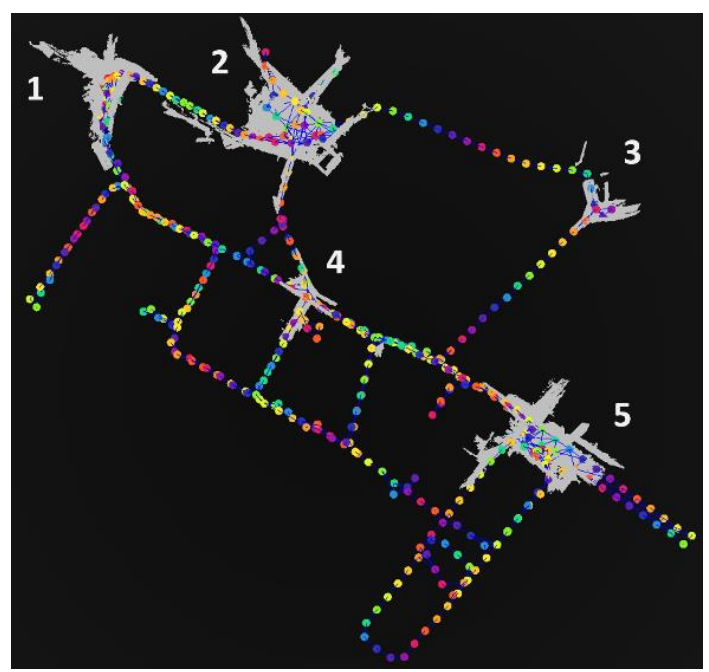

Figure 7. Planimetric view of the position of the five scans acquired with TLS Leica HDS-7000.

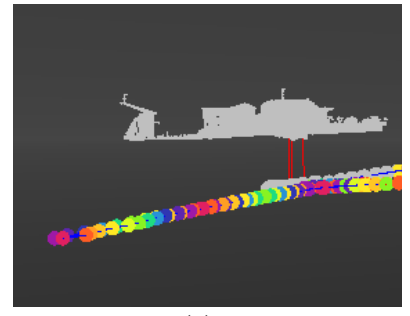

(a)

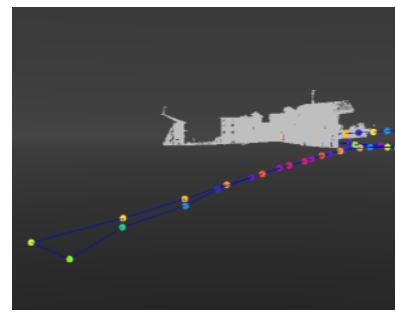

(b)
Figure 8. One of the five GCSs (grey-coloured) is introduced inside the Global Optimisation step. Comparison between the trajectory (a) before the optimisation of the cluster and (b) after the optimisation. Red segments indicate the matches error between Local Maps and GCS.

\section{RESULTS}

The accuracy of the final point clouds obtained with both the GCP and GCS methods was checked using the RTK GNSS points as Check Points (CPs) and the point cloud deriving from a photogrammetric survey performed with DJI Spark as ground truth.

Regarding the GCSs methodology, all the points were employed, while when testing the GCPs the remaining 26 points were used. As for the photogrammetric point cloud, only the portion covering Corso Matteotti and some adjacent streets were at our disposal to calculate the Cloud-to-Cloud (C2C) distance.

The accuracy check on CPs for both the methodologies reports the errors along $\mathrm{X}, \mathrm{Y}$ and $\mathrm{Z}$ directions calculated among the CPs and the corresponding points identified on the mobile mapping point cloud following the equations:

$$
\begin{aligned}
& \text { Error }_{X}=X_{G C P_{S}}-X_{I M M S} \\
& \text { Error }_{Y}=Y_{G C P_{S}}-Y_{I M M S} \\
& \text { Error }_{Z}=Z_{G C P_{S}}-Z_{I M M S}
\end{aligned}
$$

Due to the negligibility of errors along with $\mathrm{X}$ and $\mathrm{Y}$ directions, only the error along $\mathrm{Z}$ direction is reported in Figure 9 for the method involving the GCPs. For the sake of completeness and for a better comprehension of the spatial distribution, the errors along $\mathrm{X}, \mathrm{Y}$ and $\mathrm{Z}$ directions are reported in Table 2, where they are listed associated with their ID.

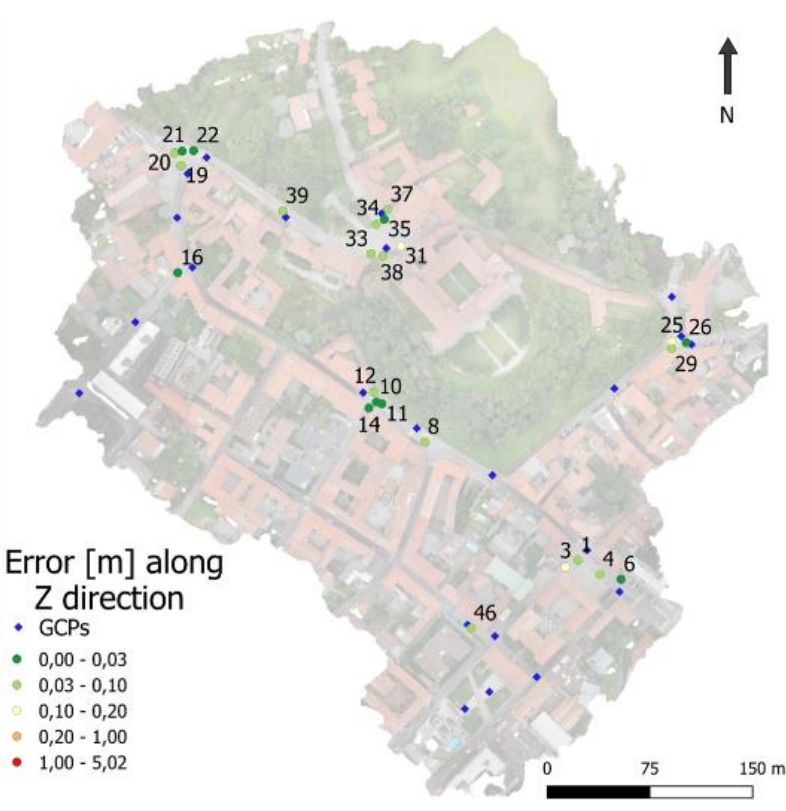

Figure 9. Accuracy check on 26 CPs when testing the GCPs method. Numbers correspond to the CPs ID. Basemap: orthophoto resulting from the photogrammetric survey performed with UAV DJI Spark.

\begin{tabular}{cccc} 
ID & $\mathbf{X}(\mathbf{m})$ & $\mathbf{Y}(\mathbf{m})$ & $\mathbf{Z}(\mathbf{m})$ \\
\hline $\mathbf{1}$ & 0.00 & 0.01 & 0.10 \\
$\mathbf{3}$ & 0.01 & 0.01 & 0.11 \\
$\mathbf{4}$ & 0.01 & 0.00 & 0.05 \\
$\mathbf{6}$ & 0.00 & 0.00 & 0.00 \\
$\mathbf{8}$ & 0.01 & 0.01 & 0.09 \\
$\mathbf{1 0}$ & 0.01 & 0.00 & 0.01 \\
$\mathbf{1 1}$ & 0.01 & 0.02 & 0.01 \\
$\mathbf{1 2}$ & 0.01 & 0.02 & 0.05 \\
$\mathbf{1 4}$ & 0.02 & 0.02 & 0.02 \\
$\mathbf{1 6}$ & 0.00 & 0.01 & 0.03 \\
$\mathbf{1 9}$ & 0.01 & 0.02 & 0.04 \\
$\mathbf{2 0}$ & 0.01 & 0.01 & 0.03 \\
$\mathbf{2 1}$ & 0.01 & 0.01 & 0.00 \\
$\mathbf{2 2}$ & 0.01 & 0.01 & 0.02 \\
$\mathbf{2 5}$ & 0.01 & 0.01 & 0.12 \\
$\mathbf{2 6}$ & 0.00 & 0.00 & 0.00 \\
$\mathbf{2 9}$ & 0.03 & 0.01 & 0.03 \\
$\mathbf{3 1}$ & 0.00 & 0.01 & 0.12 \\
$\mathbf{3 3}$ & 0.00 & 0.01 & 0.08 \\
$\mathbf{3 4}$ & 0.01 & 0.02 & 0.10 \\
$\mathbf{3 5}$ & 0.01 & 0.01 & 0.03 \\
$\mathbf{3 7}$ & 0.00 & 0.01 & 0.05 \\
$\mathbf{3 8}$ & 0.04 & 0.03 & 0.05 \\
$\mathbf{3 9}$ & 0.02 & 0.02 & 0.06 \\
$\mathbf{4 6}$ & 0.00 & 0.02 & 0.06 \\
$\mathbf{5 0}$ & 0.04 & 0.01 & 0.04 \\
\hline & & &
\end{tabular}

Table 2. Accuracy check on 26 CPs when testing the GCPs method. $\mathrm{X}, \mathrm{Y}$ and $\mathrm{Z}$ indicate the error along the homonymous direction.

The accuracy check on CPs along the $\mathrm{Z}$ direction for the method of GCSs is reported in Figure 10Figure 10. As in the previous case, errors along $\mathrm{X}$ and $\mathrm{Y}$ directions are negligible, but they are reported in Table 3 together with those along the $\mathrm{Z}$ direction. 


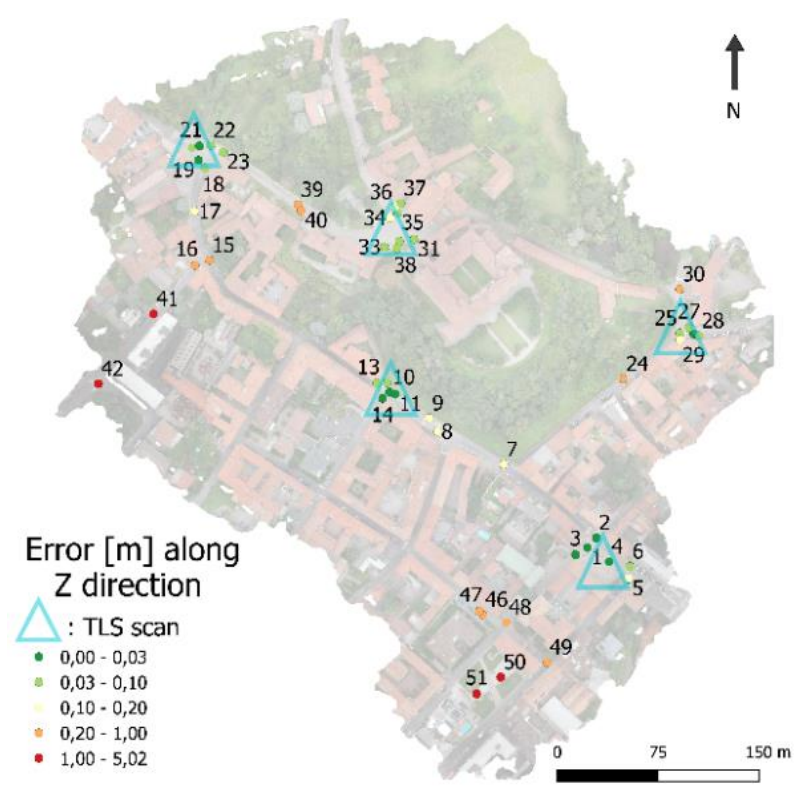

Figure 10. Accuracy check on $48 \mathrm{CPs}$ when testing the GCSs method. Numbers correspond to the CPs ID. Basemap: orthophoto resulting from the photogrammetric survey performed with UAV DJI Spark.

\begin{tabular}{cccc} 
ID & $\mathbf{X}(\mathbf{m})$ & Y(m) & $\mathrm{Z}(\mathbf{m})$ \\
\hline $\mathbf{1}$ & 0.00 & 0.00 & 0.01 \\
$\mathbf{2}$ & 0.01 & 0.00 & 0.02 \\
$\mathbf{3}$ & 0.05 & 0.01 & 0.00 \\
$\mathbf{4}$ & 0.02 & 0.01 & 0.00 \\
$\mathbf{5}$ & 0.00 & 0.01 & 0.13 \\
$\mathbf{6}$ & 0.01 & 0.00 & 0.06 \\
$\mathbf{7}$ & 0.00 & 0.00 & 0.15 \\
$\mathbf{8}$ & 0.00 & 0.00 & 0.12 \\
$\mathbf{9}$ & 0.01 & 0.00 & 0.17 \\
$\mathbf{1 0}$ & 0.02 & 0.01 & 0.01 \\
$\mathbf{1 1}$ & 0.01 & 0.00 & 0.00 \\
$\mathbf{1 2}$ & 0.01 & 0.02 & 0.07 \\
$\mathbf{1 3}$ & 0.00 & 0.01 & 0.04 \\
$\mathbf{1 4}$ & 0.00 & 0.00 & 0.01 \\
$\mathbf{1 5}$ & 0.01 & 0.01 & 0.31 \\
$\mathbf{1 6}$ & 0.00 & 0.01 & 0.55 \\
$\mathbf{1 7}$ & 0.01 & 0.01 & 0.11 \\
$\mathbf{1 8}$ & 0.01 & 0.01 & 0.05 \\
$\mathbf{1 9}$ & 0.02 & 0.01 & 0.01 \\
$\mathbf{2 0}$ & 0.00 & 0.00 & 0.05 \\
$\mathbf{2 1}$ & 0.01 & 0.01 & 0.01 \\
$\mathbf{2 2}$ & 0.00 & 0.00 & 0.03 \\
$\mathbf{2 3}$ & 0.01 & 0.01 & 0.04 \\
$\mathbf{2 4}$ & 0.02 & 0.00 & 0.48 \\
$\mathbf{2 5}$ & 0.01 & 0.02 & 0.04 \\
$\mathbf{2 6}$ & 0.02 & 0.01 & 0.00 \\
$\mathbf{2 7}$ & 0.01 & 0.00 & 0.07 \\
$\mathbf{2 8}$ & 0.04 & 0.00 & 0.03 \\
$\mathbf{2 9}$ & 0.02 & 0.00 & 0.17 \\
$\mathbf{3 0}$ & 0.02 & 0.01 & 0.56 \\
$\mathbf{3 1}$ & 0.02 & 0.00 & 0.07 \\
$\mathbf{3 2}$ & 0.00 & 0.00 & 0.04 \\
$\mathbf{3 3}$ & 0.01 & 0.00 & 0.04 \\
$\mathbf{3 4}$ & 0.03 & 0.00 & 0.14 \\
$\mathbf{3 5}$ & 0.04 & 0.01 & 0.07 \\
$\mathbf{3 6}$ & 0.00 & 0.00 & 0.11 \\
$\mathbf{3 7}$ & 0.00 & 0.00 & 0.04 \\
$\mathbf{3 8}$ & 0.00 & 0.01 & 0.05 \\
$\mathbf{3 9}$ & 0.02 & 0.01 & 0.23
\end{tabular}

\begin{tabular}{llll}
$\mathbf{4 0}$ & 0.01 & 0.01 & 0.21 \\
$\mathbf{4 1}$ & 0.01 & 0.02 & 2.14 \\
$\mathbf{4 2}$ & 0.01 & 0.00 & 5.02 \\
$\mathbf{4 6}$ & 0.01 & 0.02 & 0.61 \\
$\mathbf{4 7}$ & 0.04 & 0.01 & 0.49 \\
$\mathbf{4 8}$ & 0.02 & 0.00 & 0.53 \\
$\mathbf{4 9}$ & 0.01 & 0.02 & 0.94 \\
$\mathbf{5 0}$ & 0.02 & 0.01 & 1.30 \\
$\mathbf{5 1}$ & 0.00 & 0.02 & 1.74 \\
\hline
\end{tabular}

Table 3. Accuracy check on 48 CPs when testing the GCSs method. $\mathrm{X}, \mathrm{Y}$ and $\mathrm{Z}$ indicate the error along the homonymous direction.

The $\mathrm{C} 2 \mathrm{C}$ distances between the point cloud derived from the GCSs method, the one deriving from the GCPs method and the photogrammetric one are reported in Figure 11. Analyses were performed using CloudCompare software. For the same reason as above, the $\mathrm{C} 2 \mathrm{C}$ reported distance refers to the $\mathrm{Z}$ direction. Different sections of the three-point clouds were extracted through a polyline drawn in correspondence with the road centreline. The photogrammetric point cloud served as a reference.

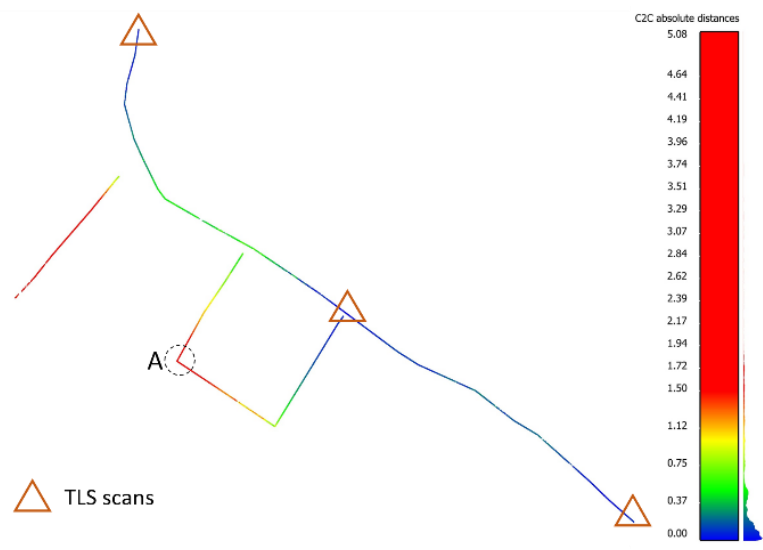

(a)

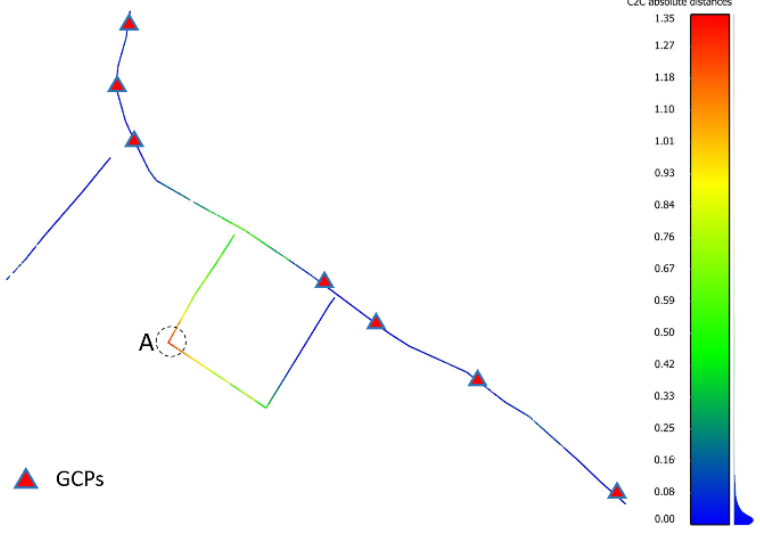

(b)

Figure 11. C2C distance along the $\mathrm{Z}$ direction (a) between the point cloud derived from the GCSs method and the photogrammetric point cloud and (b) between the point cloud derived from the GCPs method and the photogrammetric point cloud. Unit of measure: metre.

Finally, a more systematic analysis involving Corso Matteotti is performed and reported in Table 4. The analysis regards the GCPs and GCSs methods and the point cloud processed without using any constraints. Punctual comparison of the distance 
between the point clouds along $\mathrm{Z}$ direction has been performed at 49 locations equally spaced $10 \mathrm{~m}$ apart along Corso Matteotti (Figure 12).

The analysis aims at a detailed study of the error in a trajectory section i) constrained at the beginning, in the middle and at the end as far as the GCSs method is concerned, ii) constrained on 7 well-distributed points when dealing with the GCPs method and iii) not constrained at all but only georeferenced on one point at the beginning and two at the end when taking into account the third point cloud.

Also in this case, the photogrammetric point cloud was used as ground truth. The distance has been calculated following Equation 1.

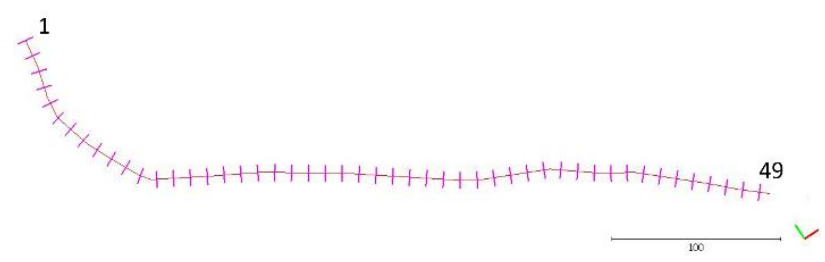

Figure 12. Subdivision of Corso Matteotti in 49 sections equally spaced $10 \mathrm{~m}$ apart along the polyline drawn in correspondence with the road centreline. Punctual comparison was performed at the intersection between the 50 polylines.

\begin{tabular}{rrrc}
\hline & $\begin{array}{c}\text { GCPs } \\
(\mathbf{m})\end{array}$ & $\begin{array}{c}\text { GCSs } \\
(\mathbf{m})\end{array}$ & $\begin{array}{c}\text { NO constraints } \\
(\mathbf{m})\end{array}$ \\
\hline $\mathbf{1}$ & 0.01 & 0.01 & 2.75 \\
$\mathbf{2}$ & 0.09 & 0.09 & 0.76 \\
$\mathbf{3}$ & 0.11 & 0.10 & -1.36 \\
$\mathbf{4}$ & 0.07 & 0.00 & -3.91 \\
$\mathbf{5}$ & 0.07 & 0.04 & -6.20 \\
$\mathbf{6}$ & 0.13 & -0.10 & -8.39 \\
$\mathbf{7}$ & 0.01 & -0.15 & -9.59 \\
$\mathbf{8}$ & -0.03 & -0.20 & -10.64 \\
$\mathbf{9}$ & 0.01 & -0.19 & -11.31 \\
$\mathbf{1 0}$ & 0.06 & -0.31 & -12.12 \\
$\mathbf{1 1}$ & -0.04 & -0.34 & -12.59 \\
$\mathbf{1 2}$ & -0.07 & -0.47 & -13.22 \\
$\mathbf{1 3}$ & -0.10 & -0.50 & -12.89 \\
$\mathbf{1 4}$ & -0.17 & -0.51 & -11.54 \\
$\mathbf{1 5}$ & -0.20 & -0.54 & -10.27 \\
$\mathbf{1 6}$ & -0.27 & -0.34 & -8.85 \\
$\mathbf{1 7}$ & -0.39 & -0.27 & -7.68 \\
$\mathbf{1 8}$ & -0.41 & -0.45 & -6.44 \\
$\mathbf{1 9}$ & -0.45 & -0.45 & -5.29 \\
$\mathbf{2 0}$ & -0.50 & -0.45 & -4.41 \\
$\mathbf{2 1}$ & -0.30 & -0.31 & -3.53 \\
$\mathbf{2 2}$ & -0.10 & -0.08 & -2.63 \\
$\mathbf{2 3}$ & -0.02 & -0.01 & -1.90 \\
$\mathbf{2 4}$ & -0.10 & -0.07 & -1.38 \\
$\mathbf{2 5}$ & 0.03 & 0.03 & -0.76 \\
$\mathbf{2 6}$ & 0.02 & 0.03 & -0.32 \\
$\mathbf{2 7}$ & 0.01 & 0.01 & 0.00 \\
$\mathbf{2 8}$ & 0.00 & -0.06 & 0.39 \\
$\mathbf{2 9}$ & 0.02 & -0.05 & 0.72 \\
$\mathbf{3 0}$ & 0.06 & -0.07 & 0.95 \\
$\mathbf{3 1}$ & 0.00 & -0.13 & 1.36 \\
$\mathbf{3 2}$ & 0.03 & -0.12 & 1.81 \\
$\mathbf{3 3}$ & 0.05 & -0.06 & 2.53 \\
$\mathbf{3 4}$ & 0.01 & -0.20 & 3.21 \\
$\mathbf{3 5}$ & 0.02 & -0.12 & 4.10 \\
$\mathbf{3 6}$ & -0.03 & -0.09 & 4.79 \\
$\mathbf{3 7}$ & -0.01 & -0.17 & 4.96 \\
$\mathbf{3 8}$ & -0.12 & -0.22 & 4.92 \\
& & &
\end{tabular}

\begin{tabular}{lrrl}
39 & -0.02 & -0.11 & 4.82 \\
$\mathbf{4 0}$ & -0.01 & -0.10 & 4.81 \\
$\mathbf{4 1}$ & -0.06 & -0.17 & 4.90 \\
$\mathbf{4 2}$ & -0.08 & -0.16 & 4.73 \\
$\mathbf{4 3}$ & -0.04 & -0.10 & 4.19 \\
$\mathbf{4 4}$ & -0.05 & -0.06 & 3.66 \\
$\mathbf{4 5}$ & -0.04 & -0.06 & 3.12 \\
$\mathbf{4 6}$ & -0.01 & -0.02 & 2.45 \\
$\mathbf{4 7}$ & -0.02 & -0.01 & 1.70 \\
$\mathbf{4 8}$ & 0.01 & 0.00 & 0.90 \\
$\mathbf{4 9}$ & -0.01 & -0.06 & 0.21 \\
\hline
\end{tabular}

Table 4. Distance between the GCPs, GCSs and point cloud without any constraints along $\mathrm{Z}$ direction, using the photogrammetric point cloud as ground truth.

Punctual comparison at 49 locations along Corso Matteotti.

\section{DISCUSSION AND CONCLUSION}

The accuracy check of the GCSs methodology using CPs (Figure 10) shows that the errors increase as moving away from the constraints, reaching up to $1.74 \mathrm{~m}$ at $\mathrm{CP} 51$ and $5 \mathrm{~m}$ at $\mathrm{CP} 42$ (Table 3). The same behaviour can be observed in Figure 11a, where the $\mathrm{C} 2 \mathrm{C}$ distance is $1.80 \mathrm{~m}$ at the crossroads marked with label A.

Results are very different when looking at GCPs methodology. In this case, the punctual check reported in Figure 9 and Table 2 shows a maximum error of $0.12 \mathrm{~m}$ in correspondence with CP 31 . Nevertheless, the comparison with the photogrammetric point cloud shown in Figure $11 \mathrm{~b}$ reveals a maximum altitude error of $1.30 \mathrm{~m}$ at the crossroads marked with label A.

However, when considering the systematic analysis reported in Table 4 , it can be observed that the maximum error is $0.54 \mathrm{~m}$ for the GCSs method - in correspondence of section 15 - and 0.50 $\mathrm{m}$ for the GCPs one - in correspondence of section 20 .

From the reported analyses and results, focusing on Corso Matteotti, it can be stated that GCPs method performed better than GCSs one. It should be noted, however, that the GCPs used as constraints were well distributed over the entire trajectory, whereas the GCSs were only at the beginning, in the middle and at the end.

To conclude, IMMS Backpack Heron MS Twin Color has allowed us to survey quickly a vast urban area not otherwise obtainable with traditional survey techniques. SLAM algorithm was crucial in this scenario, where the presence of urban canyons made it impossible to rely on MMS using GNSS positioning.

The paper demonstrated how constraints are essential to improve the trajectory reconstruction and correct the drift error, as can be deduced from the distances reported in Table 4. Looking at the last column, it can be observed that the maximum error of the trajectory without any constraints is $-13.22 \mathrm{~m}$, reached in correspondence of section 12 . It is evident that such values cannot be admitted.

Time taken to collect the scans and the GNSS points was comparable. The Ground Control Scans method was developed to understand how high redundant robust constraints, binding the mobile point cloud in all directions, introduced in the SLAM process affect the accuracy of the final trajectory and point cloud. Reported results show their key role in the procedure. It constitutes a crucial point in the architecture and cultural heritage field, where nowadays it is very easy to find existing point clouds that can be used as constraints in a more extended mobile mapping survey.

As for the use of Ground Control Points, the paper demonstrated that, despite the punctual constraint they provide, the resulting 
trajectory is significantly improved thanks to their redundancy and distribution all over the area.

Future developments will certainly investigate the different configurations of GCSs and GCPs to find the optimal spatial distribution minimising the field's processing time and operator effort.

\section{ACKNOWLEDGEMENTS}

The authors would like to report that the survey is part of a complex project to enhance the historic centre of Meda (MB), coordinated by Prof. Laura Pezzetti of the Politecnico di Milano.

\section{REFERENCES}

Cantoni, S., Vassena, G., 2019. Fast indoor mapping to feed in an indoor DB for building and facility management. ISPRS Ann. Photogramm. Remote Sens. Spat. Inf. Sci. 42, 213-217. https://doi.org/10.5194/isprs-archives-XLII-2-W9-213-2019

Gexcel official web page [WWW Document], 2022. URL https://gexcel.it/it/ (accessed 1.21.22).

Jung, J., Yoon, S., Ju, S., Heo, J., 2015. Development of kinematic 3D laser scanning system for indoor mapping and asbuilt BIM using constrained SLAM. Sensors (Switzerland) 15, 26430-26456. https://doi.org/10.3390/s151026430

Karam, S., Vosselman, G., Peter, M., Hosseinyalamdary, S., Lehtola, V., 2019. Design, calibration, and evaluation of a backpack indoor mobile mapping system. Remote Sens. 11. https://doi.org/10.3390/rs11080978

Lagüela, S., Dorado, I., Gesto, M., Arias, P., González-Aguilera, D., Lorenzo, H., 2018. Behavior analysis of novel wearable indoor mapping system based on 3d-slam. Sensors (Switzerland) 18, 1-16. https://doi.org/10.3390/s18030766

Lehtola, V. V., Kaartinen, H., Nüchter, A., Kaijaluoto, R., Kukko, A., Litkey, P., Honkavaara, E., Rosnell, T., Vaaja, M.T., Virtanen, J.P., Kurkela, M., El Issaoui, A., Zhu, L., Jaakkola, A., Hyyppä, J., 2017. Comparison of the selected state-of-the-art 3D indoor scanning and point cloud generation methods. Remote Sens. 9, 1-26. https://doi.org/10.3390/rs9080796

Marotta, F., Teruggi, S., Achille, C., Vassena, G.P.M., Fassi, F., 2021. Integrated Laser Scanner Techniques to Produce HighResolution DTM of Vegetated Territory. Remote Sens. 13. https://doi.org/10.3390/rs13132504

Nocerino, E., Menna, F., Remondino, F., Toschi, I., RodríguezGonzálvez, P., 2017. Investigation of indoor and outdoor performance of two portable mobile mapping systems. Videometrics, Range Imaging, Appl. XIV 10332, 103320I. https://doi.org/10.1117/12.2270761

Puente, I., González-Jorge, H., Martínez-Sánchez, J., Arias, P., 2013. Review of mobile mapping and surveying technologies. Meas. J. Int. Meas. Confed. 46, 2127-2145. https://doi.org/10.1016/j.measurement.2013.03.006

Tucci, G., Visintini, D., Bonora, V., Parisi, E.I., 2018. Examination of indoor mobile mapping systems in a diversified internal/external test field. Appl. Sci. 8 . https://doi.org/10.3390/app8030401
Wang, C., Wen, C., Dai, Y., Yu, S., Liu, M., 2020. Urban 3D modeling with mobile laser scanning: a review. Virtual Real.

Intell. Hardw. 2, 2, 175-212. https://doi.org/10.1016/j.vrih.2020.05.003 